\title{
Consensus Recommendations on the Use of ${ }^{18}$ F-FDG PET/CT in Lung Disease
}

Delphine L. Chen ${ }^{1}$, Safia Ballout ${ }^{2}$, Laigao Chen $^{3}$, Joseph Cheriyan ${ }^{4,5}$, Gourab Choudhury ${ }^{6}$, Ana M. Denis-Bacelar ${ }^{7}$, Elise Emond ${ }^{8}$, Kjell Erlandsson ${ }^{8}$, Marie Fisk ${ }^{5}$, Francesco Fraioli ${ }^{8}$, Ashley M. Groves ${ }^{8}$, Roger N. Gunn ${ }^{9}, 10$, Jun Hatazawa ${ }^{11}$, Beverley F. Holman ${ }^{12}$, Brian F. Hutton ${ }^{8}$, Hidehiro Iida ${ }^{13}$, Sarah Lee ${ }^{14}$, William MacNee ${ }^{6}$, Keiko Matsunaga ${ }^{11}$, Divya Mohan ${ }^{15}$, David Parr ${ }^{16}$, Alaleh Rashidnasab ${ }^{8}$, Gaia Rizzo ${ }^{9,10}$, Deepak Subramanian ${ }^{17}$, Ruth Tal-Singer ${ }^{15}$, Kris Thielemans ${ }^{8}$, Nicola Tregay ${ }^{5}$, Edwin J.R. van Beek ${ }^{6}$, Laurence Vass ${ }^{5}$, Marcos F. Vidal Melo ${ }^{18}$, Jeremy W. Wellen ${ }^{19}$, Ian Wilkinson ${ }^{4,5}$, Frederick J. Wilson ${ }^{20}$, and Tilo Winkler ${ }^{18}$

${ }^{1}$ Department of Radiology, University of Washington, Seattle Cancer Care Alliance, Seattle, Washington; ${ }^{2}$ School of Physics and Astronomy, University of Leeds, Leeds, United Kingdom; ${ }^{3}$ Worldwide Research, Development, and Medical, Pfizer Inc., Cambridge, Massachusetts; ${ }^{4}$ Cambridge University Hospitals, NHS Foundation Trust, Cambridge, United Kingdom; ${ }^{5}$ Department of Medicine, University of Cambridge, Cambridge, United Kingdom; ${ }^{6}$ Edinburgh Imaging, Queen's Medical Research Institute, University of Edinburgh, Edinburgh, United Kingdom; ${ }^{7}$ National Physical Laboratory, Teddington, United Kingdom; ${ }^{8}$ Institute of Nuclear Medicine, University College London, London, United Kingdom; ${ }^{9}$ inviCRO, London, United Kingdom; ${ }^{10}$ Department of Medicine, Imperial College London, London, United Kingdom; ${ }^{11}$ Department of Nuclear Medicine and Tracer Kinetics, Osaka University, Osaka, Japan; ${ }^{12}$ Nuclear Medicine Department, Royal Free Hospital, London, United Kingdom; ${ }^{13}$ Faculty of Biomedicine and Turku PET Center, University of Turku, Turku, Finland; ${ }^{14}$ Amallis Consulting Ltd., London, United Kingdom; ${ }^{15}$ Medical Innovation, Value Evidence, and Outcomes, GlaxoSmithKline R\&D, Collegeville, Pennsylvania; ${ }^{16}$ University Hospitals Coventry and Warwickshire, Coventry, United Kingdom; ${ }^{17}$ Derby Teaching Hospitals, NHS Foundation Trust, Derby, United Kingdom; ${ }^{18}$ Department of Anesthesia, Critical Care, and Pain Medicine, Massachusetts General Hospital and Harvard Medical School, Boston, Massachusetts; ${ }^{19}$ Research and Early Development, Celgene, Cambridge, Massachusetts; and ${ }^{20}$ Clinical Imaging, Clinical Pharmacology, and Experimental Medicine, GlaxoSmithKline, Stevenage, United Kingdom

PET with ${ }^{18} \mathrm{~F}-\mathrm{FDG}$ has been increasingly applied, predominantly in the research setting, to study drug effects and pulmonary biology and to monitor disease progression and treatment outcomes in lung diseases that interfere with gas exchange through alterations of the pulmonary parenchyma, airways, or vasculature. To date, however, there are no widely accepted standard acquisition protocols or imaging data analysis methods for pulmonary ${ }^{18} \mathrm{~F}-\mathrm{FDG}$ PET/CT in these diseases, resulting in disparate approaches. Hence, comparison of data across the literature is challenging. To help harmonize the acquisition and analysis and promote reproducibility, we collated details of acquisition protocols and analysis methods from 7 PET centers. From this information and our discussions, we reached the consensus recommendations given here on patient preparation, choice of dynamic versus static imaging, image reconstruction, and image analysis reporting.

Key Words: pulmonary imaging; FDG; PET/CT; lung disease

J Nucl Med 2020; 61:1701-1707

DOI: 10.2967/jnumed.120.244780

Received May 11, 2020; revision accepted Sep. 9, 2020.

For correspondence or reprints contact: Delphine L. Chen, University of Washington, Seattle Cancer Care Alliance, 1144 Eastlake Ave. E, LG2-200, Seattle, WA 98109.

E-mail: dlchen7@uw.edu

Published online Sep. 18, 2020.

COPYRIGHT (c) 2020 by the Society of Nuclear Medicine and Molecular Imaging.
$\mathbf{P}$ setting, as an imaging indicator of molecular changes for monitoring disease progression and treatment effects. The pathogenesis of these diseases leads to reduced gas exchange through alterations of the pulmonary parenchyma, airways, or vasculature (1-4). However, the development of effective therapies remains disappointingly slow, hampered by the limited ability to quantify molecular changes in the lungs and assess drug binding and activity. ${ }^{18} \mathrm{~F}-\mathrm{FDG}$ is the most widely available and commonly applied PET tracer and is predominantly used to assess lung inflammation and fibrosis-related processes for both research and clinical purposes. New molecularly targeted PET tracers are also being developed to support the respiratory drug development process, with a recent publication demonstrating the additional potential for PET imaging to assess drug target engagement in the lungs (5). Therefore, we anticipate that the application of PET imaging in lung diseases will continue to grow across multiple centers.

In light of this growing use, standardization of acquisition protocols and analysis methods will facilitate data comparisons from multicenter studies and across the literature. To date, a range of analysis methods has been applied to disparately acquired static and dynamic datasets in studies of lung disease and related processes (6-13). Given that the lungs uniquely contain varying amounts of air depending on the disease, are the source of respiratory motion, and have a relatively high fractional blood volume, special considerations are needed when applying quantitative imaging approaches. Because of the variation in protocols among centers, however, comparability of the results across centers is somewhat limited. 
Our group has previously published a summary of the conceptual approaches for lung PET imaging and highlighted some of the issues with clinical and research applications (2). To build on these applications, ensure comparability of data across centers, and enable cross-center data comparisons, we identified a need for harmonized protocols. Therefore, we convened representatives from all academic and commercial centers active in PET lung imaging to develop and present consensus recommendations for patient preparation, scanning protocol design, and imaging data analysis. The primary aim is to improve standardization to support uniform data collection and interpretation, thus improving the potential to compare results and pool data across research studies and enable advancement of the field. Moreover, it is hoped that a better understanding of the origin of the PET signal, gained from studies using dynamic acquisition and complex analyses, will enable the development of simpler static measurement protocols that can be more readily applied in clinical practice. Our consensus recommendations are timely given the current higher level of attention on uniform protocols to enhance the reproducibility of quantitative PET, as evidenced by recently published consensus papers on cancer and neuroimaging (14-17).

The acquisition protocols and image analysis details (Supplemental Tables 1 and 2; supplemental materials are available at http:// jnm.snmjournals.org) were collated from 7 participating centers that have conducted PET studies on lung diseases (Cambridge University Hospitals NHS Foundation Trust and University of Cambridge, Invicro, Massachusetts General Hospital, University College London, University Hospitals Coventry and Warwickshire, University of Edinburgh, and Washington University in St Louis; Supplemental Table 3). The acquisition protocol information included patient preparation, image acquisition, and image reconstruction. The analysis details included the whole and regional lung delineation methods and imaging endpoint derivations from both static and dynamic PET acquisitions. Between March 2018 and August 2019, the collated information was reviewed and the consensus recommendations developed over the course of 3 meetings that we attended in person or via teleconference. The collated details of the acquisition protocols were reviewed by all of us. Protocol differences among the centers were discussed and resolved to produce the consensus recommendations. When considering data analysis details, it was clear from the start that the desired imaging endpoints would vary among studies depending on the individual study objectives. Therefore, we defined minimum reporting requirements with a sufficient level of detail to enable comparison of published studies from different centers and to improve the reproducibility of analyses. All authors reviewed and approved the following recommendations.

\section{NOTEWORTHY}

- This consensus statement provides recommendations for ${ }^{18}$ F-FDG PET lung imaging protocols, image analysis, and reporting to facilitate comparison of data acquired at different centers.

- Corrections for the effects of air (air fraction correction) and blood feature prominently in the quantitative analysis methods that are proposed to improve lung tissue-specific ${ }^{18} \mathrm{~F}-\mathrm{FDG}$ quantification.

- Novel reconstruction methods and other approaches may help overcome the challenge of minimizing the effects of respiratory motion on quantification.

\section{PATIENT PREPARATION}

\section{Fasting Period}

To minimize glucose-related inhibition of ${ }^{18} \mathrm{~F}$-FDG uptake, only plain water should be consumed during a fasting period lasting a minimum of $6 \mathrm{~h}$ before scanning. This is also the recommendation of the Quantitative Imaging Biomarkers Alliance for oncologic PET imaging (16).

\section{Blood Glucose Level}

Among the protocols collated, the minimum acceptable blood glucose level before scanning varied. We agreed that a lower limit of $4 \mathrm{mmol} / \mathrm{L}$ would help avoid requiring glucose administration for preventing hypoglycemia that might interfere with the scan results. The European Association of Nuclear Medicine guidelines for tumor imaging (14) recommend an upper limit of $11 \mathrm{mmol} / \mathrm{L}$ for clinical studies. However, because medication such as steroids and other factors can influence glucose levels, we felt this should be a guide and that upper limits should be determined on a perstudy basis.

Similarly, regarding the fasting period and acceptable blood glucose and insulin levels for diabetic patients, there were insufficient data to allow us to make a firm recommendation. We agreed that it would be reasonable to consider excluding patients with diabetes from small studies to minimize confounding factors.

\section{PATIENT POSITIONING AND COMFORT}

For pulmonary scanning, arm placement within the field of view can cause CT attenuation artifacts that may compromise the accuracy of the PET data. Therefore, having patients' arms above their head for the duration of the scan is preferred. For patients who cannot tolerate this position, ensuring that the arms fit completely within the CT field of view consistently is recommended to reduce inaccurate attenuation correction across the chest when comparing studies. A maximum body mass index of $35 \mathrm{~kg} / \mathrm{m}^{2}$ is generally suggested to minimize both variability in positioning of patients within the scanner and body habitus effects. We also recommend that patients with implants that are within the chest field of view, such as pacemakers, be excluded from studies to avoid extreme artifacts.

Prolonged periods of lying down may be difficult for patients with lung diseases and may result in gross movement, causing attenuation correction errors. Measures should be taken to maximize comfort during scanning, including allowing a continuous supply of oxygen when needed, placing a vacuum bag filled with pillows or memory foam padding under the arms to provide support and improve blood circulation, using knee wedges to increase back comfort, and applying blankets for warmth (4). Appropriate padding of pressure points and avoidance of joint overstretching are particularly important for sedated, mechanically ventilated patients. Providing breaks during a dynamic PET scan for patients to sit or stand may be an option but will require rigorous realignment of each scan portion to produce a contiguous time-activity curve.

\section{ADMINISTERED ${ }^{18}$ F-FDG ACTIVITY}

${ }^{18} \mathrm{~F}-\mathrm{FDG}$ doses used for clinical oncologic scans as outlined in the European Association of Nuclear Medicine guidelines (14) are sufficient for a static clinical scan in most lung diseases. Since many factors determine the choice of injected activity, such as static or dynamic image acquisition, frame duration, desired 
TABLE 1

Recommendations on Patient Preparation, Acquisition Protocols, and Image Reconstruction

\begin{tabular}{|c|c|}
\hline Category & Recommendation \\
\hline \multicolumn{2}{|l|}{ Patient preparation } \\
\hline Fasting period & Minimum of $6 \mathrm{~h}$ before scanning, with plain water allowed \\
\hline Blood glucose level & Minimum of $4 \mathrm{mmol} / \mathrm{L}$, with upper limit determined by study objectives \\
\hline Patient positioning & $\begin{array}{l}\text { Arms above head when possible, with maximum body mass index of } 35 \mathrm{~kg} / \mathrm{m}^{2} \text { allowed for } \\
\text { consistent arm positioning }\end{array}$ \\
\hline $\begin{array}{l}\text { Administered }{ }^{18} \mathrm{~F}-\mathrm{FDG} \\
\text { activity and injection duration }\end{array}$ & $\begin{array}{l}\text { Activity no more than needed for sufficient image quality to meet study objectives, with injection } \\
\text { duration documented and kept consistent for all patients }\end{array}$ \\
\hline \multicolumn{2}{|l|}{ Acquisition protocol } \\
\hline Static vs. dynamic & $\begin{array}{l}\text { Static scans acceptable if compatible with study objectives; dynamic scans recommended when } \\
\text { tracer quantification for specific lung compartments is needed; rationale for chosen acquisition } \\
\text { method reported }\end{array}$ \\
\hline Respiratory gating & $\begin{array}{l}\text { End-expiration gating results reported when used; list-mode data stored to allow future } \\
\text { reprocessing as techniques improve }\end{array}$ \\
\hline $\begin{array}{l}\text { Accounting for } \\
\text { respiratory motion }\end{array}$ & $\begin{array}{l}\text { Breath-hold at end-expiration or mid-expiration most frequently used to match lung volumes for } \\
\text { CT and PET; prescan coaching of breathing instructions recommended (coaching patient on } \\
\text { breathing instructions for attenuation correction CT can minimize most respiratory motion } \\
\text { errors); exploration of approaches such as cine-CT }\end{array}$ \\
\hline \multirow[t]{3}{*}{ Duration and time frame } & Static imaging: starting at $60 \mathrm{~min}$ after injection \\
\hline & $\begin{array}{l}\text { Dynamic imaging: } 45-\text { to } 90 \text {-min acquisition starting immediately after injection; } 60 \text {-min scan is } \\
\text { routinely tolerated (breaks may be required for improved patient tolerance) }\end{array}$ \\
\hline & $\begin{array}{l}\text { Time frames for typical } 60-\text { min dynamic acquisition (time after injection): 0-2 min: 5-15 s/frame; } \\
2-5 \text { min: } 20-30 \text { s/frame; 5-10 min: } 60 \text { s/frame; } 10-18 \text { min: } 120 \text { s/frame; } 18-30 \text { min: } \\
180 \text { s/frame; } 30-60 \text { min: } 300 \text { s/frame }\end{array}$ \\
\hline Image reconstruction & $\begin{array}{l}\text { Method harmonized as much as possible in multicenter study; for iterative reconstruction, larger } \\
\text { number of iterations should be considered to ensure uniform convergence, regardless of image } \\
\text { reconstruction algorithm, followed by suitable filter to control noise if desired }\end{array}$ \\
\hline
\end{tabular}

analysis endpoints, and standard practice at the study location (supplemental materials), no recommendation can be made to fit all studies. The introduction of more sensitive PET scanners could also affect the choice of injected dose. For research studies, the minimum activity that provides sufficient image quality to meet the study objectives should be used (18), and we recommend that this activity be determined on a per-study basis.

\section{Injection Duration}

Very little information has been published on injection duration for dynamic acquisitions, even though this can influence the compartmental modeling results. Observations at several participating centers indicate that a bolus with a shorter injection duration and adequate early-frame time sampling will better characterize the peak of the blood time-activity curve and may avoid a biased estimate of

TABLE 2

Recommendations on Image Analysis Parameters

\begin{tabular}{|c|c|c|c|}
\hline Modality & Analysis method & Abbreviation & Description \\
\hline Static & Body-weight SUV $\max$ & $\mathrm{SUV}_{\max }$ & Maximum SUV* (unitless) \\
\hline \multirow[t]{4}{*}{ Dynamic } & Patlak & $K_{i}$ & Net influx rate of ${ }^{18} \mathrm{~F}-\mathrm{FDG}^{*}$ (mL plasma/mL lung/min) \\
\hline & & $V_{s s}$ & Steady-state partition coefficient $\left(\mathrm{mL} / \mathrm{cm}^{3}\right)$ \\
\hline & Compartmental modeling & $K_{i c}$ & $\begin{array}{l}\text { Net influx rate of }{ }^{18} \mathrm{~F}-\mathrm{FDG}(\mathrm{mL} \text { plasma/mL lung/min) } \\
\quad\left(\text { from } K_{1}, k_{2} \text {, and } k_{3}\right)^{*}\end{array}$ \\
\hline & & $V_{b}$ & Fractional blood volume (unitless) \\
\hline $\mathrm{CT}$ & Air fraction determination & $V_{a}$ & Fractional air volume (unitless) \\
\hline
\end{tabular}


TABLE 3

Recommendations on Minimum Compartmental Modeling Method Parameters to Be Reported

\begin{tabular}{|c|c|}
\hline Parameter & Description \\
\hline Weighting factors & How factors were calculated \\
\hline Time delay & Method used to fit time delay \\
\hline $\begin{array}{l}\text { Input function } \\
\text { modeling }\end{array}$ & $\begin{array}{l}\text { Method used to define input } \\
\text { function }\end{array}$ \\
\hline Vessel volume & $\begin{array}{l}\text { Size, position, and methods used } \\
\text { for vessel segmentation }\end{array}$ \\
\hline Lung VOls & $\begin{array}{l}\text { Method used for whole or } \\
\text { regional lung segmentation }\end{array}$ \\
\hline Goodness of fit & Method used to evaluate data fitting \\
\hline
\end{tabular}

fractional blood volume ( $V_{b}$, unitless), whereas a longer injection duration may lead to improved fitting of the lung time-activity curves and better estimate the metabolic rate. Until these observations have been validated, a specific injection duration cannot be recommended. Until then, we recommend that the injection technique be defined for each study protocol and reported accordingly, with steps taken to ensure consistency for all scans within a study, particularly when several personnel are involved.

\section{IMAGE ACQUISITION}

\section{Static Versus Dynamic}

Static PET imaging is the most common acquisition protocol used clinically. Static image endpoints have been shown to correlate with lung physiology and quality-of-life measurements (19) and thus may be sufficient to meet the study objectives. For example, static acquisitions may be sufficient for studies focused on areas of higher density, such as fibrotic regions or inflammatory nodules, that often demonstrate relatively high uptake levels. Using a reference region may also provide sufficient correction for differences in the blood signal due to systemic factors, such as variations in the metabolism of white blood cells, without the need for a full kinetic model, as explored for idiopathic pulmonary fibrosis (12). Delayed postinjection static images may also help reduce the influence of blood activity by allowing it to clear, as previously explored using images obtained at $180 \mathrm{~min}$ after injection (20). However, this delay may make quantification in normal lung tissue challenging because of the very low signal. Major advantages of static acquisitions include a short scanning duration that is well tolerated, as well as broader accessibility, as every clinical site will have the capacity to acquire and analyze the data.

Dynamic imaging enables estimation of kinetic parameters and can add insights into the origins of the PET signal. Kinetic modeling of blood and lung cell compartments (parenchyma, airway wall, vascular wall, and immune cells (2)) to enable corrections for $V_{b}$ contributions may be achievable $(11,21)$, thus making dynamic acquisitions an important consideration when attempting to account for such effects with a single tracer. Additionally, dynamic scan parameters have been shown to be more sensitive than static endpoints in quantifying the presence of low-level lung inflammation under certain conditions, such as in acute lung injury models (22), and in discriminating the sources of increased ${ }^{18} \mathrm{~F}$ FDG uptake in different mechanisms of injury (23). The aims of each research study will define the optimal approach, and the choice should be justified when the study is reported.

The partial volume of air in each voxel will affect both static and dynamic acquisitions and may vary among lung regions. This effect can be corrected using appropriately acquired CT images matched to the PET images for respiration, referred to here using the term air fraction correction. As new tracers are introduced for lung imaging, the kinetics and dynamic range of uptake will also be important factors in deciding between static and dynamic acquisitions.

\section{Respiratory Motion}

Because of the unique function of the lungs, the large variation in air volume with normal respiration can reduce the accuracy of PET quantitation. Clinical CT examinations are routinely performed during a breath-hold, often at full inspiration. Since respiration must occur during the longer static and dynamic PET scans, locally varying displacement and compression of tissues will potentially contribute to errors in both attenuation correction and air fraction correction. The displacement causes blurring and increases the partial-volume effect, particularly near the diaphragm, whereas the compression of tissues affects density and radiotracer concentration (24). A mismatch between the point in the respiratory cycle at which the CT portion of the PET/CT scan is acquired and the average position represented by the PET data can therefore lead to artifacts from attenuation correction and air fraction correction and is an important source of error.

Multiple approaches to removing or limiting respiratory motion have been investigated. Shallow breathing during the CT acquisition or breath-holds at gentle end-expiration, as for cancer imaging (25), or at mid-expiration for idiopathic pulmonary fibrosis patients $(26,27)$ can minimize the effects of misregistration of PET and CT images due to respiratory motion. Visual feedback systems to monitor and continuously display the lung volume to the subject have been used but are not widely adopted (28). Acquisitions using repeated breath-holds have also been suggested (29) but can be difficult for those with reduced lung function. Longer CT scans acquired over the duration of the respiratory cycle, such as cine-CT or low-pitch helical CT, can be used to create averaged CT scans (30). A 4-dimensional CT dataset sorted according to the respiratory signal can be used for gated PET data (31), with several studies investigating various respiratory gating strategies for PET (32). Interpolated averaged CT scans constructed from full-inspiration and end-expiration scans may also be considered (33), a method that could also be applied to CT scans obtained from dynamic imaging protocols in which breaks are built into the dynamic acquisition (34).

The approach most used among the centers to minimize PET and CT misregistration is breath-hold at gentle end-expiration or at mid-expiration for the CT scan. Prescan coaching to familiarize the patient with breathing instructions improved compliance and is thus recommended. Since many of the advanced techniques described above are not widely available and require postimaging offline processing, we recommend that, for research studies, listmode acquisitions be acquired and stored for future reprocessing when respiratory gating and other advanced techniques to minimize respiratory motion become readily available.

\section{Duration and Time Frame}

Clinical static ${ }^{18} \mathrm{~F}-\mathrm{FDG}$ PET imaging protocols have been used successfully to image lung disease (19). Typically, data are acquired over a short interval starting approximately $60 \mathrm{~min}$ after 
tracer injection. For dynamic imaging studies, acquisitions start at the same time as tracer administration and range from 45 to $90 \mathrm{~min}$ in duration. The scan durations reported by the centers are routinely tolerated without discomfort or safety issues and allow robust kinetic analysis. Typically, short frames are used initially to capture the early dynamics of the tracer in blood and are followed by longer frames later in the acquisition. For a typical 60-min scan, our recommended time frames are 5-15 s/frame for the first $2 \mathrm{~min}$ after injection, 20-30 s/frame at 2-5 min, $60 \mathrm{~s} /$ frame at 5-10 $\mathrm{min}, 120 \mathrm{~s} /$ frame at 10-18 $\mathrm{min}, 180 \mathrm{~s} /$ frame at 18-30 $\mathrm{min}$, and $300 \mathrm{~s} /$ frame at $30-60 \mathrm{~min}$. We again recommend retaining the list-mode data to allow future exploration of different time frames.

Most modern PET/CT scanners can cover the entire lung at gentle end-expiration at a single bed position. For those requiring 2 bed positions to cover the entire lung, a dynamic data acquisition initially over 1 bed position followed by a static data acquisition at 2 bed positions may be used. Alternating 2 bed positions over the scan duration, such as alternating seven 4-min acquisitions at each position, is another approach that more easily accommodates breaks between the bed-position scans. Breaks should be scheduled in such a way that disruption of acquisition of the time-activity curve peak during the vascular phase after injection is avoided (Fig. 1). The recently developed total-body PET scanner (35) will eliminate the need for such protocols but is not yet widely available.

\section{IMAGE RECONSTRUCTION}

Various PET image reconstruction algorithms are available, with different algorithms leading to different noise and image characteristics, depending on the choice of parameters, filters, or a priori assumptions (36). Further, for iterative algorithms, the convergence rate of the values in low-count regions such as the lungs is often lower than in high-count regions $(37,38)$. This difference may affect the observed radiotracer concentration in the lung; therefore, we recommend using a large number of iterative updates (i.e., number of iterations times number of subsets) to ensure uniform convergence $(38,39)$. We agreed that more investigation is needed to define optimal reconstruction specifically for lung PET

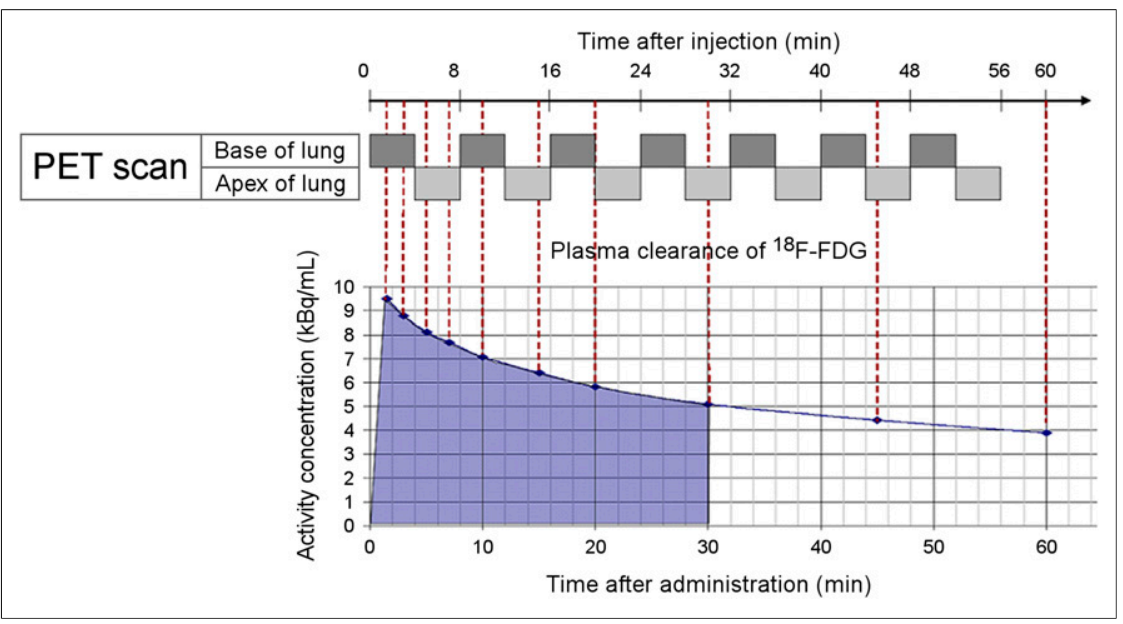

FIGURE 1. Illustration of alternating 2 bed positions over scan duration. A 4-min acquisition phase for each bed position enables data collection from lung apex and base. Blood sampling times are indicated by dashed red lines, and blood activity is indicated by the blue line. (Adapted with permission of (10).) imaging and that we therefore cannot make specific recommendations based on current data. Additionally, the development of specific phantoms that model relevant aspects of lung physiology and enable the establishment of harmonization standards will be needed to support multicenter studies.

Time-of-flight (TOF) PET scanners have been shown to reduce noise, especially in large patients (40). In addition, the local effects of attenuation mismatch between reconstructed non-TOF PET and CT images are reduced (41-43), albeit at the expense of nonlocal effects outside the lung (27). Although TOF PET/CT scanners have become more widely available, insufficient data are available to make a firm recommendation on whether TOF is valuable for quantitative lung imaging. For consistent results in multicenter studies, we recommend that either data from scanners with a similar TOF time resolution be used (44) or that only nonTOF reconstructed images be used.

Table 1 summarizes our recommendations on patient preparation, acquisition protocols, and image reconstruction.

\section{IMAGE ANALYSIS REPORTING}

Quantitative PET pulmonary image analysis involves extracting volumes of interest (VOIs) and quantifying the ${ }^{18} \mathrm{~F}-\mathrm{FDG}$ signal within the VOIs. As different study requirements preclude an absolute recommendation of a single methodology for all future studies, we agreed that consistent reporting, in accordance with the guidelines below, would enable better comparison of studies and interpretation of findings from future studies.

\section{Whole Lung Analysis}

Use of the attenuation correction CT for lung segmentation is recommended. For consistency, performing a quality control check on the segmented lung mask is essential to exclude the chest wall, major airways, bullae, heart, and liver. The lung mask created in CT space should be resampled to PET resolution, for which we recommend using the nearest-neighbor method to obtain a binary mask suitable for VOI processing. In the presence of any gross movement of the patient during the PET and CT acquisitions, the images should be registered to minimize the differences. Details should be provided on methods to confirm that the lung mask segmentation PET and CT are aligned.

\section{Regional Lung Analysis}

To investigate regional ${ }^{18} \mathrm{~F}-\mathrm{FDG}$ distribution, the whole lung mask can be subdivided into multiple regions either by anatomic lobes, such as through fissure detection (45), or by simple geometric division into upper, middle, and lower lung zones based on either length or volume $(10,11)$. Similarly, anterior-posterior subdivisions and regions with different densities have also been used. There is insufficient information to allow recommendation of a single optimal approach, as the approach may also vary with the disease. Therefore, we recommend reporting all regional analysis details, including definition and selection of VOI location and size. 


\section{Quantification of ${ }^{18}$ F-FDG Uptake}

Static and dynamic analysis techniques have been investigated to account for various lung-specific issues in PET quantification as described above. Below, we describe our consensus recommendations for reporting these quantities.

Static Data Analysis. SUV ${ }_{\max }$ (unitless), defined as the highest single-voxel value within a VOI, is the most widely used measure of ${ }^{18} \mathrm{~F}-\mathrm{FDG}$ uptake and warrants investigation despite several potential confounding factors that could influence its measurement, including partial-volume-averaging effects (15). Corrections to the SUV for fractional air volume ( $V_{a}$, unitless) and $V_{b}$, when independently measured, should be applied and reported (46,47). SUV peak, without or with correction for lean body mass (unitless), measured from an approximately $1 \mathrm{~cm}^{3}$ VOI with the highest value as defined by PERCIST (48), is less influenced by image noise and may also be considered. Comparing areas of diseased lung to normal lung to determine a target-to-background ratio (unitless), as well as characterizing lung heterogeneity, may also be useful approaches $(12,49)$.

Dynamic Data Analysis. Dynamic datasets can be analyzed using either Patlak graphical analysis or compartmental modeling. In both cases, an input function is required to describe the time course of radioactivity concentration in arterial plasma. An image-derived input function may be obtained from the dynamic PET data in preference to blood sampling. Image-derived input functions from the pulmonary artery, ascending aorta, descending aorta, and superior vena cava have been investigated (50). In our combined experience, the use of the pulmonary artery, ascending aorta, and descending aorta reached similar outcomes for rate of transfer of ${ }^{18} \mathrm{~F}$-FDG (influx constant, or $K_{i}$ [mL plasma/mL lung/min]) $(51,52)$. The right ventricle can also be used as a source for the image-derived input function (53) but is not useful if not consistently included in the field of view for lung PET scans. Time delays between passage of the tracer through the image-derived input function VOI and the tissue of interest may affect blood volume estimates but are less important for $K_{i}$. The size of the VOI should be reported and should be consistent for all patients within the same study.

Patlak Graphical Analysis. Patlak graphical analysis estimates the net influx rate of irreversibly trapped tracers such as ${ }^{18} \mathrm{~F}-\mathrm{FDG}$ from the blood into target tissue $\left(K_{i}\right)$ and an approximate steady-state partition coefficient between tissue and plasma of nonphosphorylated ${ }^{18} \mathrm{~F}-\mathrm{FDG}$ $\left(V_{s s}, \mathrm{~mL} / \mathrm{cm}^{3}\right)$ (54). $V_{s s}$ was initially assumed to reflect changes in density, leading to explorations of normalizing $K_{i}$ with $V_{s s}$ (6). However, normalizing the $K_{i}$ by the Patlak intercept has been shown algebraically to remain sensitive to changes in $V_{a}$ and $V_{b}(11,21)$. Therefore, caution is advised in its application and interpretation as a measure of the true net influx rate. For accurate comparison, the results of Patlak graphical analysis should include both $K_{i}$ and $V_{s s}$ individually if normalized $K_{i}$ is used, as reported previously $(6,10-12)$.

Compartmental Modeling. Nonlinear regression models are used to estimate the microparameters of the model $\left(K_{1}[\mathrm{~mL}\right.$ plasma/mL lung/min], $k_{2}$ [1/min], $k_{3}$ [1/min]) and the blood contribution $\left(V_{b}\right)$ from lung time-activity curves and the imagederived input function, to then estimate $K_{i}$ using a 2-compartment model (2). Since ${ }^{18}$ F-FDG can be considered irreversibly trapped over the time frame of the scan, $k_{4}$ is assumed to be zero. Air fraction correction may also be included in the compartmental modeling, for which net influx rate is denoted as $K_{i c}(11,50)$. The quality of the fit of the data should be assessed and reported using a metric such as $\chi^{2}$. Care should be taken to report the compartmental modeling method accurately to enable reproducibility, given the various approaches available. The presence of edema in some conditions such as acute lung injury may require an additional compartment (55).

Table 2 summarizes reporting of image analysis parameters. Table 3 lists compartmental modeling-specific parameters that we recommend to promote reproducibility in reporting (56).

\section{CONCLUSION}

To improve reproducibility both between and within centers and the potential for data pooling, we urge investigators using PET/CT in studies of lung disease to follow the recommendations presented in this article when designing, conducting, and reporting studies. As highlighted in this article, ample opportunities for investigation exist to improve the methods used to acquire and analyze lung PET/CT images. We hope this summary will serve as a basis for advancements in the field of lung PET imaging.

\section{DISCLOSURE}

The Institute of Nuclear Medicine at UCL receives support from the NIHR University College London Hospitals Biomedical Research Centre. The National Physical Laboratory receives funding from the National Measurement System program of the U.K.'s Department of Business, Energy, and Industrial Strategy. Joseph Cheriyan receives funding from the Cambridge NIHR Comprehensive Biomedical Research Centre and GlaxoSmithKline. Edwin van Beek is supported by the Scottish Imaging Network (SINAPSE). Laurence Vass receives funding from EPSRC. Delphine Chen receives funding from the National Institutes of Health (R01 HL121218, R01 HL131908, and P41 EB025815). Laigao Chen is an employee and shareholder of Pfizer, Inc. Joseph Cheriyan is obliged to spend $50 \%$ of NHS time for GlaxoSmithKline. Brian Hutton, Kris Thielemans, and Kjell Erlandsson receive funding from GlaxoSmithKline, GE Healthcare, Siemens Healthineers, and Kromek. Alaleh Rashidnasab and Elise Emond receive funding from GlaxoSmithKline. Sarah Lee is an employee and the company director of Amallis and receives consulting fees from GE Healthcare and GlaxoSmithKline. Divya Mohan and Ruth Tal-Singer are former employees of GlaxoSmithKline. Divya Mohan is a current employee and shareholder of Genentech/Roche. Frederick Wilson is an employee and shareholder of GlaxoSmithKline. Ruth Tal-Singer is a shareholder of GlaxoSmithKline and receives consulting fees from Immunomet, Vocalis Health, and Ena Therapeutics. Gaia Rizzo is an employee of inviCRO. Edwin van Beek is the owner of QCTIS Ltd, is on the advisory boards of Aidence and Imbio, and receives funding from Siemens, GE Healthcare, and Alliance Medical. Marcos Vidal Melo receives funding from NIH-NHLBI RO1 HL121228. Laurence Vass receives funding from GSK (STU100043346 and BIDS3000023795). Jeremy Wellen is an employee and shareholder of Celgene. No other potential conflict of interest relevant to this article was reported.

\section{ACKNOWLEDGMENT}

We acknowledge the late Martin Connell for his tremendous contributions to the PET in Lung Diseases Collaboration Initiative and his dedication to the field of quantitative medical imaging analysis.

\section{REFERENCES}

1. Scherer PM, Chen D. Imaging pulmonary inflammation. J Nucl Med. 2016;57: 1764-1770.

2. Chen DL, Cheriyan J, Chilvers ER, et al. Quantification of lung PET images: challenges and opportunities. J Nucl Med. 2017;58:201-207. 
3. Lukey PT, Harrison SA, Yang S, et al. A randomised, placebo-controlled study of omipalisib (PI3K/mTOR) in idiopathic pulmonary fibrosis. Eur Respir J. 2019; 53:1801992.

4. Chen DL, Huang HJ, Byers DE, et al. The peroxisome proliferator-activated receptor agonist pioglitazone and 5-lipoxygenase inhibitor zileuton have no effect on lung inflammation in healthy volunteers by positron emission tomography in a single-blind placebo-controlled cohort study. PLoS One. 2018;13:e0191783.

5. Maher TM, Simpson JK, Porter JC, et al. A positron emission tomography imaging study to confirm target engagement in the lungs of patients with idiopathic pulmonary fibrosis following a single dose of a novel inhaled $\alpha v \beta 6$ integrin inhibitor. Respir Res. 2020;21:75.

6. Jones HA, Sriskandan S, Peters AM, et al. Dissociation of neutrophil emigration and metabolic activity in lobar pneumonia and bronchiectasis. Eur Respir J. 1997;10: 795-803.

7. Chen DL, Rosenbluth DB, Mintun MA, Schuster DP. FDG-PET imaging of pulmonary inflammation in healthy volunteers after airway instillation of endotoxin. J Appl Physiol. 2006;100:1602-1609.

8. Chen DL, Bedient TJ, Kozlowski J, et al. $\left[{ }^{18} \mathrm{~F}\right]$ fluorodeoxyglucose positron emission tomography for lung antiinflammatory response evaluation. Am J Respir Crit Care Med. 2009;180:533-539.

9. Harris RS, Venegas JG, Wongviriyawong C, et al. ${ }^{18} \mathrm{~F}-\mathrm{FDG}$ uptake rate is a biomarker of eosinophilic inflammation and airway response in asthma. $\mathrm{J} \mathrm{Nucl}$ Med. 2011;52:1713-1720.

10. Subramanian DR, Jenkins L, Edgar R, Quraishi N, Stockely R, Parr D. Assessment of pulmonary neutrophilic inflammation in emphysema by quantitative positron emission tomography. Am J Respir Crit Care Med. 2012;186:1125-1132.

11. Coello C, Fisk M, Mohan D, et al. Quantitative analysis of dynamic ${ }^{18}$ F-FDG PET/CT for measurement of lung inflammation. EJNMMI Res. 2017;7:47.

12. Win T, Screaton NJ, Porter JC, et al. Pulmonary ${ }^{18}$ F-FDG uptake helps refine current risk stratification in idiopathic pulmonary fibrosis (IPF). Eur J Nucl Med Mol Imaging. 2018;45:806-815.

13. Motta-Ribeiro GC, Hashimoto S, Winkler T, et al. Deterioration of regional lung strain and inflammation during early lung injury. Am J Respir Crit Care Med. 2018;198:891-902.

14. Boellaard R, Delgado-Bolton R, Oyen WJG, et al. FDG PET/CT: EANM procedure guidelines for tumour imaging-version 2.0. Eur J Nucl Med Mol Imaging. 2015;42:328-354.

15. Kinahan PE, Perlman ES, Sunderland JJ, et al. The QIBA profile for FDG PET/ $\mathrm{CT}$ as an imaging biomarker measuring response to cancer therapy. Radiology. 2020;294:647-657.

16. FDG-PET/CT Technical Committee. QIBA Profile: FDG-PET/CT as an Imaging Biomarker Measuring Response to Cancer Therapy-Version 1.13. Radiological Society of North America; November 2016.

17. Knudsen GM, Ganz M, Appelhoff S, et al. Guidelines for the content and format of PET brain data in publications and archives: a consensus paper. J Cereb Blood Flow Metab. 2020;40:1576-1585.

18. International Commission on Radiological Protection. ICRP publication 62: radiological protection in biomedical research. Ann ICRP. 1992;22.

19. Groves AM, Win T, Screaton NJ, et al. Idiopathic pulmonary fibrosis and diffuse parenchymal lung disease: implications from initial experience with ${ }^{18} \mathrm{~F}-\mathrm{FDG}$ PET/CT. J Nucl Med. 2009;50:538-545.

20. Umeda Y, Demura Y, Morikawa M, et al. Prognostic value of dual-time-point ${ }^{18} \mathrm{~F}$ FDG PET for idiopathic pulmonary fibrosis. J Nucl Med. 2015;56:1869-1875.

21. Holman BF, Cuplov V, Millner L, et al. Improved correction for the tissue fraction effect in lung PET/CT imaging. Phys Med Biol. 2015;60:7387-7402.

22. Chen DL, Mintun MA, Schuster DP. Comparison of methods to quantitate ${ }^{18} \mathrm{~F}-$ FDG uptake with PET during experimental acute lung injury. J Nucl Med. 2004;45:1583-1590.

23. de Prost N, Feng Y, Wellman T, et al. ${ }^{18}$ F-FDG kinetics parameters depend on the mechanism of injury in early experimental acute respiratory distress syndrome. J Nucl Med. 2014;55:1871-1877.

24. Cuplov V, Holman B, McClelland J, Modat M, Hutton B, Thielemans K. Issues in quantification of registered respiratory gated PET/CT in the lung. Phys Med Biol. 2017;63:015007.

25. Gilman MD, Fischman AJ, Krishnasetty V, Halpern EF, Aquino SL. Optimal CT breathing protocol for combined thoracic PET/CT. AJR. 2006;187:1357-1360.

26. Holman BF, Cuplov V, Hutton B, Groves A, Thielemans K. The effect of respiratory induced density variations on non-TOF PET quantitation in the lung. Phys Med Biol. 2016;61:3148-3163.

27. Emond EC, Bousse A, Machado M, et al. Effect of attenuation mismatches in time of flight PET reconstruction. Phys Med Biol. 2020;65:085009.

28. Harris RS, Winkler T, Musch G, et al. The prone position results in smaller ventilation defects during bronchoconstriction in asthma. J Appl Physiol. 2009;107: 266-274.
29. Skretting A, Revheim M, Knudtsen I, Johnsrud K, Bogsrud T. An implementation of time-efficient respiratory-gated PET acquisition by repeated breath-holds. Acta Radiol. 2013;54:672-675.

30. Pan T, Mawlawi O, Luo D, et al. Attenuation correction of PET cardiac data with low-dose average CT in PET/CT. Med Phys. 2006;33:3931-3938.

31. Moorees J, Bezak E. Four dimensional CT imaging: a review of current technologies and modalities. Australas Phys Eng Sci Med. 2012;35:9-23.

32. Bettinardi V, De Bernardi E, Presotto L, Gilardi M. Motion-tracking hardware and advanced applications in PET and PET/CT. PET Clin. 2013;8:11-28.

33. Mok GSP, Sun T, Tzung-Chi Huang, Vai MI. Interpolated average CT for attenuation correction in PET: a simulation study. IEEE Trans Biomed Eng. 2013;60:1927-1934.

34. Holman BF, Cuplov V, Millner L, et al. Improved quantitation and reproducibility in multi-PET/CT lung studies by combining CT information. EJNMMI Phys. 2018;5:14.

35. Cherry SR, Jones T, Karp JS, Qi J, Moses WW, Badawi RD. Total-body PET: maximizing sensitivity to create new opportunities for clinical research and patient care. J Nucl Med. 2018;59:3-12.

36. Defrise M, Kinahan PE, Michel CJ. Image reconstruction algorithms in PET. In: Bailey DL, Townsend DW, Valk PE, Maisey MN, eds. Positron Emission Tomography. Springer-Verlag; 2005:63-91.

37. Hutton BF, Hudson HM, Beekman FJ. A clinical perspective of accelerated statistical reconstruction. Eur J Nucl Med. 1997;24:797-808.

38. Boellaard R, van Lingen A, Lammertsma A. Experimental and clinical evaluation of iterative reconstruction (OSEM) in dynamic PET: quantitative characteristics and effects on kinetic modeling. J Nucl Med. 2001;42:808-817.

39. Jaskowiak CJ, Bianco JA, Perlman SB, Fine JP. Influence of reconstruction iterations on ${ }^{18}$ F-FDG PET/CT standardized uptake values. J Nucl Med. 2005;46:424-428.

40. Watson CC. An evaluation of image noise variance for time-of-flight PET. IEEE Trans Nucl Sci. 2007;54:1639-1647.

41. Turkington TG, Wilson JM. Attenuation artifacts and time-of-flight PET. In: 2009 IEEE Nuclear Science Symposium Conference Record (NSS/MIC). IEEE; 2009:2997-2999.

42. Ahn S, Cheng L, Manjeshwar RM. Analysis of the effects of errors in attenuation maps on PET quantitation in TOF PET. In: 2014 IEEE Nuclear Science Symposium and Medical Imaging Conference (NSS/MIC). IEEE; 2014:1-4.

43. Thielemans K, Asma E, Manjeshwar RM, Ganin A, Spinks TJ. Image-based correction for mismatched attenuation in PET images. In: 2008 IEEE Nuclear Science Symposium Conference. IEEE; 2008:5292-5296.

44. Kotasidis FA, Mehranian A, Zaidi H. Impact of time-of-flight on indirect 3D and direct 4D parametric image reconstruction in the presence of inconsistent dynamic PET data. Phys Med Biol. 2016;61:3443-3471.

45. Pu J, Zheng B, Leader JK, et al. Pulmonary lobe segmentation in CT examinations using implicit surface fitting. IEEE Trans Med Imaging. 2009;28:1986-1996.

46. Lambrou T, Groves AM, Erlandsson K, et al. The importance of correction for tissue fraction effects in lung PET: preliminary findings. Eur J Nucl Med Mol Imaging. 2011;38:2238-2246.

47. Valind SO, Rhodes CG, Brudin LH, Jones T. Measurements of regional ventilation pulmonary gas volume: theory and error analysis with special reference to positron emission tomography. J Nucl Med. 1991;32:1937-1944.

48. Wahl RL, Jacene H, Kasamon Y, Lodge MA. From RECIST to PERCIST: evolving considerations for PET response criteria in solid tumors. J Nucl Med. 2009;50(suppl):122S-150S

49. Winkler T, Vidal Melo MF, Degani-Costa LH, et al. Estimation of noise-free variance to measure heterogeneity. PLoS One. 2015;10:e123417.

50. Holman BF. Improving Quantification of PET/CT Biomarkers for Evaluation of Disease Progression and Treatment Effectiveness in Pulmonary Fibrosis. $\mathrm{PhD}$ thesis. University College London; 2016.

51. van der Weerdt AP, Klein LJ, Boellaard R, Visser CA, Visser FC, Lammertsma AA. Image-derived input functions for determination of MRGlu in cardiac ${ }^{18} \mathrm{~F}$-FDG PET scans. J Nucl Med. 2001;42:1622-1629.

52. de Geus-Oei L-F, Visser EP, Krabbe PFM, et al. Comparison of image-derived and arterial input functions for estimating the rate of glucose metabolism in therapy-monitoring ${ }^{18}$ F-FDG PET studies. J Nucl Med. 2006;47:945-949.

53. Schuster DP, Kaplan J, Gauvain K, Welch M, Markham J. Measurement of regional pulmonary blood flow with PET. J Nucl Med. 1995;36:371-377.

54. Patlak CS, Blasberg RG, Fenstermacher JD. Graphical evaluation of blood-tobrain transfer constants from multiple-time uptake data. J Cereb Blood Flow Metab. 1983;3:1-7.

55. Schroeder T, Vidal Melo MF, Musch G, Harris RS, Venegas JG, Winkler T. Modeling pulmonary kinetics of 2-deoxy-2-[18 F $]$ fluoro-D-glucose during acute lung injury. Acad Radiol. 2008;15:763-775.

56. Vass LD, Lee S, Wilson FJ, Fisk M, Cheriyan J, Wilkinson I. Reproducibility of compartmental modelling of ${ }^{18} \mathrm{~F}$-FDG PET/CT to evaluate lung inflammation. EJNMMI Phys. 2019;6:26. 